TRANSACTIONS OF THE

AMERICAN MATHEMATICAL SOCIETY

Volume 365, Number 7, July 2013, Pages 3397-3411

S 0002-9947(2012)05591-7

Article electronically published on December 12, 2012

\title{
ON TRIVIALITY OF THE EULER CLASS GROUP OF A DELETED NEIGHBOURHOOD OF A SMOOTH LOCAL SCHEME
}

\author{
MRINAL KANTI DAS \\ Dedicated to Professor S. M. Bhatwadekar on his 65th birthday
}

\begin{abstract}
Let $(R, \mathfrak{m})$ be a regular local ring of dimension $d$ which is essentially of finite type over a field $k$ such that the residue field of $R$ is infinite. Let $f \in \mathfrak{m} \backslash \mathfrak{m}^{2}$ be a regular parameter and $n$ be an integer such that $2 n \geq d+1$. Let $I \subset R_{f}$ be an ideal of height $n$ such that $I / I^{2}$ is generated by $n$ elements. It is proved that any given set of $n$ generators of $I / I^{2}$ can be lifted to a set of $n$ generators of $I$.
\end{abstract}

\section{INTRODUCTION}

Let $k$ be an infinite perfect field and let $X=\operatorname{Spec} R$, where $R$ is the localization of a $k$-algebra $B$ of finite type with respect to a regular prime ideal $\mathfrak{p} \in \operatorname{Spec} B$. Let $D$ be a divisor on $X$ with a decomposition $D=\bigcup_{i=1}^{t} D_{i}$ into its irreducible components $D_{i}$ such that each $D_{i}$ is non-singular and they intersect transversally. Consider $Y=X-D$, a deleted neighbourhood of the closed point $x_{0}$ of $X$. Let $E$ be a vector bundle on $Y$. In this setup, $E$ is trivial in the following cases: (i) $\operatorname{dim}(X) \leq 3$ (Gabber Ga1]); (ii) $t=1$ (Bhatwadekar-Rao [BR]); (iii) $\operatorname{rank} E \geq$ $\min \left\{\left[\frac{\operatorname{dim} R}{2}\right], t\right\}$ (Rao [Ra1]).

The above exposition is borrowed from a paper of Nisnevich $\mathrm{Ni}$, which contains some beautiful applications of these 'purity theorems'. In the same setup, now let $y \in Y$ be a closed point and let $m_{y}$ be the corresponding maximal ideal of $\mathcal{O}_{Y, y}$. It is natural to ask whether $m_{y}$ is a complete intersection. Observing that the conormal bundle $m_{y} / m_{y}^{2}$ is trivial, we consider a more general situation: Let $I$ be the defining ideal of a closed subscheme $Z$ of $Y$ such that the conormal bundle $I / I^{2}$ (defined on $Z$ ) is trivial. Is $I$ a complete intersection? The questions we are addressing in this paper are a little stronger in nature: Can a given basis of $I / I^{2}$ be lifted to a minimal set of generators of I?

We remark that with the hypothesis on $X$, each component $D_{i}$ is defined by a single equation $f_{i}$ which vanishes at the closed point $x_{0}$ of $X$. Let $\mathfrak{m}$ be the maximal ideal at $x_{0}$. The condition of transversality of the intersection of $D_{i}$ 's is equivalent to saying that the images of $f_{1}, \cdots, f_{t}$ in the $R / \mathfrak{m}$-vector space $\mathfrak{m} / \mathfrak{m}^{2}$ are linearly independent. Keeping this discussion in mind, we now give an excerpt of our main results in commutative algebraic terms (see Theorem 4.2 and Corollaries 4.3 and 4.4).

Received by the editors October 15, 2010 and, in revised form, March 25, 2011

2010 Mathematics Subject Classification. Primary 13C10, 19A15, 13H05, 13 B40.

(C)2012 American Mathematical Society

Reverts to public domain 28 years from publication 
Theorem 1.1. Let $(R, \mathfrak{m})$ be a regular local ring of dimension $d$ which is essentially of finite type over a field $k$ (not necessarily perfect) such that the residue field of $R$ is infinite. Let $f_{1}, \cdots, f_{t} \in R$ be part of a regular system of parameters of $R$. Let $n$ be an integer such that $2 n \geq d+1$ and $I \subset R_{f_{1} \cdots f_{t}}$ be an ideal of height $n$. Suppose it is given that $I=\left(a_{1}, \cdots, a_{n}\right)+I^{2}$. Then, there exist $b_{1}, \cdots, b_{n}$ such that $I=\left(b_{1}, \cdots, b_{n}\right)$ with $b_{i}-a_{i} \in I^{2}$ in the following cases:

(1) $t=1$;

(2) $n=d-1$ and $1 \leq t \leq d$;

(3) in general, $n \geq \max \left\{\frac{d+1}{2}, t+2\right\}$.

Our quest began with the case $t=1$ and goes back to the celebrated paper of Quillen $\mathrm{Qu}$. Let $(R, \mathfrak{m})$ be a regular local ring and $f \in \mathfrak{m} \backslash \mathfrak{m}^{2}$ be a regular parameter. Quillen asked whether all finitely generated projective $R_{f}$-modules are free. Although the question is still open, it has an affirmative answer for equicharacteristic regular local rings, thanks to the works of Gabber [Ga1], Bhatwadekar-Rao [BR], and Popescu [Sw2. Motivated by this phenomenon, we started wondering whether the $n$-th Euler class groups $E^{n}\left(R_{f}\right)$ are trivial, where $2 n \geq \operatorname{dim}\left(R_{f}\right)+3$. Readers familiar with the definition of the Euler class groups will realize that Theorem 1.1(1) precisely affirms that. Then we proved that the 'top' Euler class groups $E^{d-1}\left(R_{f_{1} \cdots f_{t}}\right)$ are also trivial for $1 \leq t \leq d$ (statement (2) above). The general result is somewhat restricted in the sense that we need $t \leq n-2$. In the latter part of Section 4 we observe that the above results can be easily extended to regular semilocal rings (with infinite residue fields) essentially of finite type over an infinite perfect field.

We shall urge readers unfamiliar with the Euler class groups to take a quick glance at (2.8), which is just enough to follow the above discussion. Also, we have consciously presented our results and proofs in a way so that a prior knowledge of the Euler class groups is not necessary to understand most of this article.

The method of proof of our main results follows the 'standard' technique of reducing the question to probably the simplest case when $R$ is the localization of a polynomial ring $K\left[Z_{1}, \cdots, Z_{d}\right]$ over a field $K$, at the maximal ideal $\left(Z_{1}, Z_{2}, \cdots, \phi\left(Z_{d}\right)\right)$, where $\phi \in K\left[Z_{d}\right]$ is a monic irreducible polynomial, and the regular parameters are the coordinate functions. Lindel [Li] used this technique to prove the famous BassQuillen conjecture in the geometric case. To achieve our goal, we need a refined version of Lindel's method as available in Nashier [Na] (see Section 2 for a detailed statement). Similar reductions can be seen in application in two of the papers mentioned above ([BR, Ra1]), and also in the paper of Mandal-Varma [MV].

In $\mathrm{Du}$, Dutta improved Nashier's result to the case when the regular local ring is smooth and essentially of finite type over an excellent discrete valuation ring (DVR), and in Section 5 we use Dutta's result to prove (5.2), a theorem similar to (1.1) for such rings.

\section{Preliminaries}

All the rings considered in this paper are commutative and Noetherian. By the dimension of a ring we mean the Krull dimension, which is assumed to be finite. Modules are assumed to be finitely generated.

Definition 2.1 (Analytic isomorphism). Let $\phi: S \longrightarrow R$ be a homomorphism of rings and $f \in S$ be a non-zerodivisor such that: $(1) \phi(f) \in R$ is a non-zerodivisor; 
(2) $S / f S \simeq R / \phi(f) R$, induced by $\phi$. Then $S$ is the fibre product of $S_{f}$ and $R$ over $R_{f}$, and we say that $S \stackrel{\phi}{\longrightarrow} R$ is analytically isomorphic along $f$.

Example 2.2. Let $R$ be a ring and $f, g \in R$ be non-zerodivisors such that $f R+$ $g R=R$. Then $R \longrightarrow R_{g}$ is analytically isomorphic along $f$.

Example 2.3. Let $(R, \mathfrak{m})$ be a local ring. A monic polynomial $F \in R[X]$ is called a Weierstrass polynomial if $F=X^{n}+a_{1} X^{n-1}+\cdots+a_{n}$, where $a_{i} \in \mathfrak{m}$ for $1 \leq i \leq n$. It can be easily checked (see $[\mathrm{Na}, 1.7]$ for a proof) that $R[X] \hookrightarrow R[X]_{(\mathfrak{m}, X)}$ is an analytic isomorphism along $F$.

We will need the following easy lemma. For a proof the reader may refer to $\mathrm{Na}$.

Lemma 2.4. Let $K$ be an infinite field and $F \in K\left[X_{1}, \cdots, X_{n}\right]$ be a non-zero homogeneous polynomial. Then there is a homogeneous change of variables which transforms $F$ to a monic polynomial (up to a unit) in one of the variables.

Remark 2.5. In fact one can prove more generally (as shown in the proof of $\mathbb{N a}$, 1.9]) that if $R$ is a local ring and $F \in R\left[X_{1}, \cdots, X_{n}\right]$ is a non-zero form which represents a unit in $R$, then there is a homogeneous change of variables which transforms $F$ to a monic polynomial (up to a unit) in one of the variables. We shall need this observation later.

We are now going to state a theorem of Nashier which is crucial to this paper. The following form is implicit in [Na, Proof of Theorem 2.8].

Theorem 2.6 ( $[\mathrm{Na}])$. Let $(R, \mathfrak{m})$ be a regular local ring of dimension d which is essentially of finite type over a perfect field $k$. Let $g$ be any element of $\mathfrak{m}^{2}$. Let $\left(g, f_{1}, \cdots, f_{t}\right)$ be a sequence in $R$ with $f_{1}, \cdots, f_{t}$ part of a minimal set of generators of $\mathfrak{m}$ modulo $\mathfrak{m}^{2}$, where $1 \leq t \leq d-1$. Then there exists a field $K \supseteq k$ and a regular local ring $S$ (subring of $R$ ) such that

(1) $S=K\left[Z_{1}, \cdots, Z_{d}\right]_{\left(Z_{1}, \cdots, Z_{d-1}, \phi\left(Z_{d}\right)\right)}$, where $\phi\left(Z_{d}\right) \in K\left[Z_{d}\right]$ is an irreducible monic polynomial.

(2) We may assume that $Z_{i}=f_{i}$ for $1 \leq i \leq t$.

(3) $S \hookrightarrow R$ is analytically isomorphic along $h$, for some $h \in g R \cap S$.

Moreover, if $R$ has infinite residue field, then $K$ is also infinite.

The following result, due to Mandal [Ma], is crucial to this paper.

Theorem 2.7 ([Ma, Theorem 1.2]). Let $A$ be a commutative Noetherian ring and $I \subset A[T]$ be an ideal containing a monic polynomial. Suppose it is given that $I=\left(f_{1}, \cdots, f_{r}\right)+I^{2}$, where $r \geq \operatorname{dim}(A[T] / I)+2$. Then there exist $g_{1}, \cdots, g_{r}$ such that $I=\left(g_{1}, \cdots, g_{r}\right)$, where $g_{i}-f_{i} \in I^{2}$ for $1 \leq i \leq r$.

Let $A$ be a commutative Noetherian ring of dimension $d \geq 2$. The definition of the $d$-th Euler class group $E^{d}(A)$ can be found in BS2. Let $n$ be an integer such that $2 n \geq d+3$. For the definition of the $n$-th Euler class group $E^{n}(A)$, we request the reader to refer to $\mathrm{BS3}$. We only record the following remark on the Euler class groups which is relevant to this paper.

Remark 2.8. Let $A$ be a commutative Noetherian ring of dimension $d$ and let $n$ be an integer such that $2 n \geq d+3$. Then each element of $E^{n}(A)$ can be represented by a pair $\left(I, \omega_{I}\right)$, where $I \subset A$ is an ideal of height $n$ and $\omega_{I}$ is a surjection $\omega_{I}:(A / I)^{n} \rightarrow I / I^{2}$. For any such pair, $\left(I, \omega_{I}\right)=0$ in $E^{n}(A)$ if and only if $\omega_{I}$ has a lift to a surjection $\theta: A^{n} \rightarrow I$. A similar result holds in the case $n=d=2$. 


\section{ON GABBER'S THEOREM}

The following result is an analogue of Gabber's theorem Ga1. The proof is easy, and we only require the ring $R$ to be any regular local ring of dimension 3 . We may recall that Gabber proved that if $(R, \mathfrak{m})$ is a regular local ring and $f \in \mathfrak{m} \backslash \mathfrak{m}^{2}$, then all projective $R_{f}$-modules are free.

Theorem 3.1. Let $(R, \mathfrak{m})$ be a regular local ring of dimension 3 and $f \in \mathfrak{m} \backslash \mathfrak{m}^{2}$ be a regular parameter. Let $I \subset R_{f}$ be an ideal of height 2 such that $I=\left(a_{1}, a_{2}\right)+I^{2}$. Then there exist $b_{1}, b_{2}$ such that $I=\left(b_{1}, b_{2}\right)$, where $a_{i}-b_{i} \in I^{2}$ for $i=1,2$.

Proof. Applying [BS2, Lemma 2.11] we can find $e \in I^{2}$ such that $I=\left(a_{1}, a_{2}, e\right)$, where $e(1-e) \in\left(a_{1}, a_{2}\right)$. Therefore, $I_{1-e}=\left(a_{1}, a_{2}\right)$. Consider two surjections: $\alpha:\left(R_{f(1-e)}\right)^{2} \rightarrow I_{1-e}$, given by $\alpha((1,0))=a_{1}, \alpha((0,1))=a_{2}$ and $\beta:\left(R_{f e}\right)^{2} \rightarrow$ $I_{e}\left(=R_{f e}\right)$, given by $\beta((1,0))=1, \beta((0,1))=0$. Note that $\left(a_{1}, a_{2}\right)$ is a unimodular row in $R_{f e(1-e)}$. As any unimodular row of length two can be completed to a $2 \times 2$ matrix of determinant 1 , a standard patching argument implies that there is a projective $R_{f}$-module $P$ of rank 2 such that $P$ maps onto $I$. By Gabber's theorem $P$ is free. Therefore, $I$ is generated by two elements, say $I=\left(h_{1}, h_{2}\right)$.

Moreover, by Gabber's theorem it follows that stably free $R_{f}$-modules of rank 2 are free. In other words, $R_{f}^{2}$ is cancellative. Since $\operatorname{dim} R_{f}=2$, we may now appeal to [Bh, Lemma 3.2] to conclude that there exist $b_{1}, b_{2}$ such that $I=\left(b_{1}, b_{2}\right)$, where $a_{i}-b_{i} \in I^{2}$ for $i=1,2$.

Remark 3.2. The above proof actually establishes a more general result: Let $A$ be any ring (not necessarily regular) of dimension 2 such that every projective $A$ module of rank 2 is free. Then given any ideal $J \subset A$ of height 2 and a surjection $\alpha: A^{2} \rightarrow J / J^{2}$, there exists a surjection $\theta: A^{2} \rightarrow J$ which lifts $\alpha$.

Example 3.3. Both Gabber's theorem and Theorem 3.1 are not true if we invert an arbitrary $f \in R$. To see this, we consider a standard example. Let $R=$ $\mathbb{R}[X, Y, Z]_{(X, Y, Z)}$ and take $f=X^{2}+Y^{2}+Z^{2}$. Note that $(X, Y, Z)$ is a unimodular row over $R_{f}$, and therefore it defines a stably free $R_{f}$-module $P$ of rank 2 . As the tangent bundle to the real 2-sphere is not free, it can be deduced (see [Sw1, 2.4]) that $P$ is not free (equivalently, $P$ does not have a unimodular element). Now let $\alpha: P \rightarrow J$ be a surjective $R_{f}$-linear map where $J \subset R_{f}$ is an ideal of height 2 (such an $\alpha$ exists by a theorem due to Eisenbud-Evans [EE]). Let $\bar{\alpha}: P / J P \rightarrow J / J^{2}$ be the surjection induced by $\alpha$. Fix an isomorphism $\chi: R_{f} \simeq \wedge^{2}(P)$ and choose an isomorphism $\sigma:\left(R_{f} / J\right)^{2} \simeq P / J P$ such that $\bigwedge^{2}(\sigma)=\chi \otimes R_{f} / J$ (note that $R_{f} / J$ is zero dimensional). If we write $\omega_{J}=\bar{\alpha} \sigma:\left(R_{f} / J\right)^{2} \rightarrow J / J^{2}$, then the Euler class of $P$ is $e(P, \chi)=\left(J, \omega_{J}\right)$ in $E^{2}\left(R_{f}\right)$ (for the definition of the Euler class, see BS2]). Since $P$ does not have any unimodular element, by [BS2, Corollary 4.4] we have $e(P, \chi) \neq 0$ and therefore by $\left[\mathrm{BS} 2\right.$, Theorem 4.2] it follows that $\omega_{J}$ cannot be lifted to a surjection $\theta: R_{f}^{2} \rightarrow J$. This shows that (3.1) does not hold for $J$. One can further ask whether every ideal of height 2 in $R_{f}$ is at least a complete intersection (as we can see that $P$ maps onto complete intersection ideals, namely, $(Y, Z))$. Even that is not true. To see this, consider the set of all the ideals $I$ of $R_{f}$ of height 2, each of which is a surjective image of $P$ ( $P$ as above). If each of these ideals is a complete intersection, then since $R_{f}$ is an essentially affine domain over $\mathbb{R}$, it follows from $[\mathrm{BS2}$, Theorem 5.9] that $P$ has a unimodular element. This is a 
contradiction. Therefore, there exists an ideal $I$ of $R_{f}$ of height 2 such that $I / I^{2}$ is generated by 2 elements but $I$ is not 2 -generated.

We shall now derive some consequences of Gabber's theorem and Theorem 3.1. Before proceeding, the following remark is in order.

Remark 3.4. Let $(R, \mathfrak{m})$ be a regular local ring of dimension 3 and let $f \in \mathfrak{m} \backslash \mathfrak{m}^{2}$. Consider a projective $R_{f}[T]$-module $P$. Let $\mathfrak{n}$ be a maximal ideal of $R_{f}$. Then $\left(R_{f}\right)_{\mathfrak{n}}=R_{\mathfrak{n}}$ is a regular local ring of dimension 2 , and therefore by a result of Murthy [Mu,$P \otimes R_{\mathfrak{n}}[T]$ is a free $R_{\mathfrak{n}}[T]$-module. By Quillen's local global principle it follows that $P$ is extended from $R_{f}$. Now applying Gabber's theorem we conclude that $P$ is a free $R_{f}[T]$-module.

Let $(S, \mathfrak{m})$ be a regular local ring of dimension 2. By $[\mathrm{Mu}$ any projective $S[T]$ module is free. Let $I \subset S[T]$ be an ideal of height 2 such that $I=\left(f_{1}, f_{2}\right)+I^{2}$. Then it is easy to see that $I$ is generated by 2 elements. We now prove the following corollary, which is a version of [BS1, 3.16] for arbitrary regular domains. The main idea of the proof is taken from Da1, 7.1].

Corollary 3.5. Let $R$ be a regular domain of dimension 2 and $I \subset R[T]$ be an ideal of height 2 such that $h t I(0) \geq 2$ and $I=\left(F_{1}, F_{2}\right)+\left(I^{2} T\right)$. Then there exist $H_{1}, H_{2} \in I$ and $\theta \in S L_{2}(R[T] / I)$ such that: (1) $I=\left(H_{1}, H_{2}\right)$; (2) $\left(\overline{F_{1}}, \overline{F_{2}}\right) \theta=$ $\left(\overline{H_{1}}, \overline{H_{2}}\right)$, where bar denotes modulo $I^{2}$; and (3) $H_{i}(0)=F_{i}(0)$ for $i=1,2$.

Proof. Again, it follows easily using a standard patching argument that there is a projective $R[T]$-module $P$ of rank 2 with trivial determinant mapping onto $I$. Let $\alpha: P \rightarrow I$ be the surjection. Fix an isomorphism $\chi: R[T] \simeq \bigwedge^{2} P$. Since $P / I P$ is free, $\alpha$ and $\chi$ induce a set of generators of $I / I^{2}$, say $I=\left(G_{1}, G_{2}\right)+I^{2}$.

It follows from $[\mathrm{Bh}, 2.2]$ that there is a matrix $\bar{\sigma} \in G L_{2}(R[T] / I)$ with determinant (say) $\bar{F}$ such that $\left(\overline{F_{1}}, \overline{F_{2}}\right)=\left(\overline{G_{1}}, \overline{G_{2}}\right) \bar{\sigma}$. Now applying [BS2, 2.7, 2.8], we see that there exists a projective $R[T]$-module $P_{1}$ of rank 2 having trivial determinant, a trivialization $\chi_{1}$ of $\bigwedge^{2} P_{1}$, and a surjection $\beta: P_{1} \rightarrow I$ such that if the set of generators of $I / I^{2}$ induced by $\beta$ and $\chi_{1}$ is $\overline{H_{1}}, \overline{H_{2}}$, then $\left(\overline{H_{1}}, \overline{H_{2}}\right)=\left(\overline{G_{1}}, \overline{G_{2}}\right) \bar{\delta}$, where $\bar{\delta} \in G L_{2}(R[T] / I)$ has determinant $\bar{F}$. Therefore, it follows that the two sets of generators, $\left(\overline{F_{1}}, \overline{F_{2}}\right)$ and $\left(\overline{H_{1}}, \overline{H_{2}}\right)$ of $I / I^{2}$, are connected by a matrix in $S L_{2}(R[T] / I)$.

Now as $R$ is regular, $P_{1}$ is extended from $R$, say, $P_{1} \simeq Q_{1}[T]$. Since $Q_{1}$ maps onto $I(0)$, we see that if $I(0)=R$, then $Q_{1}$ is free and consequently $P_{1}$ is free. In the next paragraph we show that $P_{1}$ is also free when $I(0)$ is proper.

As $\operatorname{dim}(R)=2$, we can talk about the Euler classes over the ring $R$. Let $\chi_{1}(0): R \simeq \bigwedge^{2}\left(Q_{1}\right)$ be induced by $\chi_{1}$ and let $\omega_{I}:(R[T] / I)^{2} \rightarrow I / I^{2}$ be the surjection corresponding to the generators $\left(\overline{H_{1}}, \overline{H_{2}}\right)$. Let $\omega_{I(0)}$ denote the surjection from $(R / I(0))^{2} \rightarrow I(0) / I(0)^{2}$ induced by $\omega_{I}$. From the above discussion it is clear that $e\left(Q_{1}, \chi_{1}(0)\right)=\left(I(0), \omega_{I(0)}\right)$ in $E^{2}(R)$. Since $\left.\widetilde{\left(F_{1}(0)\right.}, \widetilde{F_{2}(0)}\right)$ and $\left.\widetilde{\left(H_{1}(0)\right.}, \widetilde{H_{2}(0)}\right)$ (here $\sim$ denotes modulo $\left.I(0)^{2}\right)$ are connected by a matrix in $S L_{2}(R / I(0)$ ), they induce the same local orientation $\omega_{I(0)}$ of $I(0)$. But we note that $I(0)=\left(F_{1}(0), F_{2}(0)\right)$, and therefore $\omega_{I(0)}$ is a global orientation (see Remark 2.8). In other words, $\left(I(0), \omega_{I(0)}\right)=0$ in $E^{2}(R)$. Therefore, it follows from BS2, Corollary 4.4] that $Q_{1}$ has a unimodular element. Since the determinant of $Q_{1}$ is free, it follows that $Q_{1}$ is free and consequently $P_{1}$ is free. This proves (1) and (2). 
To prove (3), note that $I(0)=\left(F_{1}(0), F_{2}(0)\right)=\left(H_{1}(0), H_{2}(0)\right)$ and there is some $\gamma \in S L_{2}(R / I(0))$ such that $\left.\widetilde{\left(F_{1}(0)\right.}, \widetilde{F_{2}(0)}\right)=\left(\widetilde{H_{1}(0)}, \widetilde{H_{2}(0)}\right) \gamma$. Applying BS2, Lemma 2.3], we get $\Gamma \in S L_{2}(R)$ such that $\left(F_{1}(0), F_{2}(0)\right)=\left(H_{1}(0), H_{2}(0)\right) \Gamma$. Changing $\left(H_{1}, H_{2}\right)$ by this $\Gamma$, we get the desired set of generators of $I$.

Corollary 3.6. Let $(R, \mathfrak{m})$ be a regular local ring of dimension 3 and $f \in \mathfrak{m} \backslash \mathfrak{m}^{2}$. Let $I \subset R_{f}[T]$ be an ideal of height 2 such that htI $(0) \geq 2$. Suppose it is given that $I=\left(F_{1}, F_{2}\right)+I^{2}$. Then there exist $H_{1}, H_{2} \in I$ such that: (a) $I=\left(H_{1}, H_{2}\right)$; (b) there exists $\theta \in S L_{2}\left(R_{f}[T] / I\right)$ such that $\left(\overline{F_{1}}, \overline{F_{2}}\right) \theta=\left(\overline{H_{1}}, \overline{H_{2}}\right)$, where bar denotes modulo $I^{2}$; and (c) $F_{i}(0)-H_{i}(0) \in I(0)^{2}$ for $i=1,2$.

Proof. We have $I(0)=\left(F_{1}(0), F_{2}(0)\right)+I(0)^{2}$. We first assume that $I(0)$ is a proper ideal. By Theorem 3.1 it follows that there exists $a_{1}, a_{2} \in I(0)$ such that $I(0)=\left(a_{1}, a_{2}\right)$, where $a_{i}-F_{i}(0) \in I(0)^{2}$ for $i=1,2$. Applying [BS1, 3.9] we can find $G_{1}, G_{2} \in I$ such that $I=\left(G_{1}, G_{2}\right)+\left(I^{2} T\right)$, where $G_{i}-F_{i} \in I^{2}$. If $I(0)=R$, then we can also find such $G_{1}, G_{2}$.

Since $R_{f}$ is a regular domain of dimension 2, it follows from Corollary 3.5 that there exist $H_{1}, H_{2} \in I$ and $\theta \in S L_{2}\left(R_{f}[T] / I\right)$ such that

(1) $I=\left(H_{1}, H_{2}\right)$.

(2) $\left(\overline{G_{1}}, \overline{G_{2}}\right) \theta=\left(\overline{H_{1}}, \overline{H_{2}}\right)$, where bar denotes modulo $I^{2}$.

(3) $H_{i}(0)=G_{i}(0)$ for $i=1,2$.

It is easy to see that $\left(H_{1}, H_{2}\right)$ is the desired set of generators for $I$.

\section{MAin Results}

In this section we prove our main results. In view of Theorem 3.1, we may assume that the dimension of the ring $R$ in Theorem 4.2 is at least 4 . Then $\operatorname{dim} R_{f_{1} \cdots f_{t}} \geq 3$, and for the integer $n$ below we have $n \geq 3$.

Definition 4.1. Let $k$ be a field. A regular local ring $R$ is called a regular $k$-spot if it is the localization of an affine $k$-algebra $B$ with respect to a regular prime ideal $\mathfrak{p} \in \operatorname{Spec} B$.

Theorem 4.2. Let $(R, \mathfrak{m})$ be a regular $k$-spot of dimension $d$ with infinite residue field and $n$ be an integer such that $2 n \geq d+1$. Let $f_{1}, \cdots, f_{t} \in R$ be regular parameters of $R$ (where $t \leq n-2$ ), which are linearly independent modulo $\mathfrak{m}^{2}$. Let $I \subset R_{f_{1} \cdots f_{t}}$ be an ideal of height $n$ such that $I=\left(a_{1}, \cdots, a_{n}\right)+I^{2}$. Then there exist $b_{1}, \cdots, b_{n} \in I$ such that $I=\left(b_{1}, \cdots, b_{n}\right)$, where $b_{i}-a_{i} \in I^{2}$ for $1 \leq i \leq n$. (In other words, the $n$-th Euler class group $E^{n}\left(R_{f_{1} \cdots f_{t}}\right)$ is trivial.)

Proof. We divide the proof of the theorem into a few steps.

Step 1. Following an argument similar to Swan's, as given in [Li], we first reduce the problem to the case when $k$ is perfect.

We may assume that $R=C_{\mathfrak{p}}$, where $C \simeq k\left[X_{1}, \cdots, X_{m}\right] /\left(g_{1}, \cdots, g_{l}\right)$ and $\mathfrak{p} \in$ $\operatorname{Spec}(C)$. We have $I=\left(a_{1}, \cdots, a_{n}\right)+I^{2}$. By clearing denominators if necessary, we may assume that $a_{1}, \cdots, a_{n} \in k\left[X_{1}, \cdots, X_{m}\right]$. It is easy to see that there exists $e \in I^{2}$ such that $I=\left(a_{1}, \cdots, a_{n}, e\right)$ and $e(1-e) \in\left(a_{1}, \cdots, a_{n}\right)$. Let us assume that $e(1-e)=\lambda_{1} a_{1}+\cdots+\lambda_{n} a_{n}$, where $\lambda_{i} \in R_{f_{1} \cdots f_{t}}$.

Now let $k_{0}$ be the prime field of $k$. As in Swan's argument (see [Li]), there exists a finitely generated field extension $k^{\prime}$ of $k_{0}$ contained in $k$ and a regular $k^{\prime}$-spot 
$\left(R^{\prime}, \mathfrak{m}^{\prime}\right)$ containing $f_{1}, \cdots, f_{t}, g_{1}, \cdots, g_{l}, a_{1}, \cdots, a_{n}, e, \lambda_{1}, \cdots, \lambda_{n}$ such that

(1) $R^{\prime} \hookrightarrow R$ and $\operatorname{dim} R^{\prime}=\operatorname{dim} R$.

(2) $f_{1}, \cdots, f_{t} \in R^{\prime}$ are regular parameters of $R^{\prime}$ which are linearly independent modulo $\mathfrak{m}^{\prime 2}$.

Consider the ideal $I^{\prime}=\left(a_{1}, \cdots, a_{n}, e\right)$ in $R_{f_{1} \cdots f_{t}}^{\prime}$. Note that the relation $e(1-e)=$ $\lambda_{1} a_{1}+\cdots+\lambda_{n} a_{n}$ is retained in $R_{f_{1} \cdots f_{t}}^{\prime}$. Therefore,

$$
I^{\prime}=\left(a_{1}, \cdots, a_{n}, e-\lambda_{1} a_{1}-\cdots-\lambda_{n} a_{n}\right)=\left(a_{1}, \cdots, a_{n}, e^{2}\right)
$$

which shows that $I^{\prime}=\left(a_{1}, \cdots, a_{n}\right)+I^{\prime 2}$. We also note that $I^{\prime} R_{f_{1} \cdots f_{t}}=I$. To prove the theorem, it is enough to prove that there exist $b_{1}, \cdots, b_{n} \in I^{\prime}$ such that $b_{i}-a_{i} \in I^{\prime 2}$.

We may further note that $R^{\prime}$ is also a regular $k_{0}$-spot. Therefore, from the above discussion we may assume that $k$ is perfect to start with.

Step 2. Consider $I \cap R$. Since ht $(I \cap R)=n \geq t+2$, we can find an element $g \in(I \cap R) \cap \mathfrak{m}^{2} \backslash\left(f_{1}, \cdots, f_{t}\right) R$. Since $f_{1}, \cdots, f_{t}$ are linearly independent modulo $\mathfrak{m}^{2}$, we have $\left(f_{1}, \cdots, f_{t}, g\right)$, a regular sequence. Since $R$ is local, it follows that $\left(g, f_{1}, \cdots, f_{t}\right)$ is also a regular sequence. As $k$ is perfect, by (2.6), there exists a field $K \supset k$ and a regular $K$-spot $S=K\left[Z_{1}, \cdots, Z_{d}\right]_{\left(Z_{1}, Z_{2}, \cdots, \phi\left(Z_{d}\right) \text { ) }\right.}$ (where $\phi\left(Z_{d}\right) \in K\left[Z_{d}\right]$ is an irreducible monic polynomial) such that $S \hookrightarrow R$ is analytically isomorphic along $h$ for some $h \in g R \cap S$. Moreover, $Z_{i}=f_{i}$ for $1 \leq i \leq t$.

Therefore, $S_{Z_{1} \cdots Z_{t}} \hookrightarrow R_{f_{1} \cdots f_{t}}$ is analytically isomorphic along $h$. Let $I_{1}=$ $I \cap S_{Z_{1} \cdots Z_{t}}$. By properties of analytic isomorphism it follows that $S_{Z_{1} \cdots Z_{t}} / I_{1} \simeq$ $R_{f_{1} \cdots f_{t}} / I$, and since $h \in I$, we have $I_{1} / I_{1}^{2} \simeq I / I^{2}$. Therefore, corresponding to the given set of generators of $I / I^{2}$, we have a set of generators of $I_{1} / I_{1}^{2}$. Calling them $\alpha_{1}, \cdots, \alpha_{n}$, we have:

$$
I_{1}=\left(\alpha_{1}, \cdots, \alpha_{n}\right)+I_{1}^{2} .
$$

Clearly, since $I_{1} R_{f_{1} \cdots f_{t}}=I$, it is enough to show that there exist $\beta_{1}, \cdots, \beta_{n} \in I_{1}$ such that $I_{1}=\left(\beta_{1}, \cdots, \beta_{n}\right)$, where $\beta_{i}=\alpha_{i}$ modulo $I_{1}^{2}$ for $1 \leq i \leq n$.

Step 3. Consider the ideal $J_{1}=I_{1} \cap K\left[Z_{t}, \cdots, Z_{d-1}\right]$. Then $J_{1}$ has height $\geq$ $n-t \geq 2$. Therefore, by [Na, Proposition 1.11], it contains a non-zero form $F$ in $Z_{t}, \cdots, Z_{d-1}$. We may assume that $Z_{t}$ does not divide $F$ in $K\left[Z_{t}, \cdots, Z_{d-1}\right]$. (Suppose $F=Z_{t}^{l} G$. Note that $G$ is also a form. As $F$ and $G$ are associates in $S_{Z_{1} \cdots Z_{t}}$, it follows that $G \in I_{1}$ and therefore $G \in I_{1} \cap K\left[Z_{t}, \cdots, Z_{d-1}\right]$, and we can work with $G$.) Let $F=F_{1}+Z_{t} F_{2}$, where $F_{1} \in K\left[Z_{t+1}, \cdots, Z_{d-1}\right]$ is a non-zero form.

Since the residue field of $R$ is infinite, it follows that $K$ is infinite (see Theorem 2.6). Therefore, $F_{1}$ represents a unit and we may make a homogeneous change of variables (see Lemma 2.4), involving only $Z_{t+1}, \cdots, Z_{d-1}$, and assume that, up to a unit, $F$ is a monic polynomial in some variable $Z_{i} \in\left\{Z_{t+1}, \cdots, Z_{d-1}\right\}$ with all its coefficients in $K\left[Z_{t}, \cdots, \widehat{Z_{i}}, \cdots, Z_{d-1}\right]$. (Here 'hat' over a variable means dropping that variable.) We note that $F$ remains homogeneous and that $F$ still belongs to $I_{1}$. 
Without loss of any generality, we assume that $F$ is monic in $Z_{t+1}$. If we write $A=K\left[Z_{1}, \cdots, \widehat{Z}_{t+1}, \cdots, Z_{d}\right]_{\left(Z_{1}, \cdots, \widehat{Z}_{t+1}, \cdots, \phi\left(Z_{d}\right)\right)}$, then $A$ is a local ring with maximal ideal $m=\left(Z_{1}, \cdots, \widehat{Z}_{t+1}, \cdots, \phi\left(Z_{d}\right)\right)$ and $F \in A\left[Z_{t+1}\right]$ is a Weierstrass polynomial. Therefore we have an analytic isomorphism (see Example 2.3)

$$
A\left[Z_{t+1}\right] \hookrightarrow A\left[Z_{t+1}\right]_{\left(m, Z_{t+1}\right)}
$$

along $F$. But $A\left[Z_{t+1}\right]_{\left(m, Z_{t+1}\right)}=S$.

We further obtain an analytic isomorphism $A_{Z_{1} \cdots Z_{t}}\left[Z_{t+1}\right] \hookrightarrow S_{Z_{1} \cdots Z_{t}}$, which is induced by the one above.

Recall that we have $I_{1}=\left(\alpha_{1}, \cdots, \alpha_{n}\right)+I_{1}^{2}$ and we wanted to prove that there exist $\beta_{1}, \cdots, \beta_{n} \in I_{1}$ such that $I_{1}=\left(\beta_{1}, \cdots, \beta_{n}\right)$, where $\beta_{i}=\alpha_{i}$ modulo $I_{1}^{2}$ for $1 \leq$ $i \leq n$. Now let $I_{2}=I_{1} \cap A_{Z_{1} \ldots Z_{t}}\left[Z_{t+1}\right]$. By properties of analytic isomorphism, we have $A_{Z_{1} \cdots Z_{t}}\left[Z_{t+1}\right] /(F) \simeq S_{Z_{1} \cdots Z_{t}} /(F)$, and since $F \in I_{1}$, we have $I_{1} / I_{1}^{2} \simeq I_{2} / I_{2}^{2}$. Therefore, corresponding to $\alpha_{1}, \cdots, \alpha_{n}$ we have a set of generators of $I_{2} / I_{2}^{2}$, say, given by

$$
I_{2}=\left(\mathfrak{a}_{1}, \cdots, \mathfrak{a}_{n}\right)+I_{2}^{2},
$$

and it is enough to prove that there exist $\mathfrak{b}_{1}, \cdots, \mathfrak{b}_{n} \in I_{2}$ such that $I_{2}=\left(\mathfrak{b}_{1}, \cdots, \mathfrak{b}_{n}\right)$ with $\mathfrak{b}_{i}=\mathfrak{a}_{i}$ modulo $I_{2}^{2}$. Here we note that $I_{2} \subset A_{Z_{1} \cdots Z_{t}}\left[Z_{t+1}\right]$ and $\operatorname{dim}\left(A_{Z_{1} \cdots Z_{t}}\right)=$ $d-2($ as $\operatorname{dim}(A)=d-1)$. Since $I_{2}$ contains a monic polynomial and $2 n \geq d+1$, a theorem of Mandal (2.7) ensures that we can find such $\mathfrak{b}_{1}, \cdots, \mathfrak{b}_{n}$. This finishes the proof.

Taking $t=1$ in the above theorem we obtain the following corollary, which is an analogue of Quillen's question discussed in the introduction.

Corollary 4.3. Let $(R, \mathfrak{m})$ be a regular $k$-spot of dimension $d$ with infinite residue field. Let $f \in \mathfrak{m} \backslash \mathfrak{m}^{2}$ be a regular parameter. Let $n$ be an integer such that $2 n \geq d+2$. Then the $n$-th Euler class group $E^{n}\left(R_{f}\right)$ is trivial.

In the case $n=d-1 \geq 3$, we do not need any restriction on $t$, as the following corollary shows.

Corollary 4.4. Let $(R, \mathfrak{m})$ be a regular $k$-spot of dimension $d$ with infinite residue field. Let $f_{1}, \cdots, f_{t} \in R$ be regular parameters of $R$ (where $1 \leq t \leq d$ ), which are linearly independent modulo $\mathfrak{m}^{2}$. Then,

(1) The $(d-1)$-th Euler class group $E^{d-1}\left(R_{f_{1} \cdots f_{t}}\right)$ is trivial.

(2) The Chow group of zero cycles $C H_{0}\left(R_{f_{1} \cdots f_{t}}\right)$ is trivial.

(3) The weak Euler class group $E_{0}\left(R_{f_{1} \cdots f_{t}}\right)$ is trivial.

Proof. To prove (1), we only recall a general result on Euler class groups which states that if $B$ is any commutative Noetherian ring (not necessarily regular or an affine algebra) of dimension $r$ and if $S$ is any multiplicatively closed subset of $B$ such that $\operatorname{dim}(B)=\operatorname{dim}\left(S^{-1} B\right)$, then the canonical map $E^{r}(B) \longrightarrow E^{r}\left(S^{-1} B\right)$ is a surjective group homomorphism.

As both $C H_{0}\left(R_{f_{1} \cdots f_{t}}\right)$ and $E_{0}\left(R_{f_{1} \cdots f_{t}}\right)$ are surjective images of the Euler class group $E^{d-1}\left(R_{f_{1} \cdots f_{t}}\right),(2)$ and (3) follow trivially from (1). (For the definition of the weak Euler class group, see [BS2].)

We now deduce that any zero-dimensional ideal in $R_{f_{1} \cdots f_{t}}$ is a set-theoretic complete intersection. We first need to recall the definition of a reduction of an ideal. 
Definition 4.5. Let $R$ be a ring. Let $J \subseteq I$ be ideals. $J$ is said to be a reduction of $I$ if there exists a non-negative integer $t$ such that $I^{t+1}=J I^{t}$.

Corollary 4.6. Let $k$ be an infinite field and $(R, \mathfrak{m})$ be a regular $k$-spot of dimension $d \geq 3$. Let $f_{1}, \cdots, f_{t}$ be regular parameters and $I \subset R_{f_{1} \cdots f_{t}}$ be an ideal. We write $A=R_{f_{1} \cdots f_{t}}$. We have the following assertions.

(1) If $\operatorname{dim}(A / I)=0$, then $I$ is a set-theoretic complete intersection.

(2) If $\operatorname{dim}(A / I)=1$ and $I$ is a local complete intersection, then $I$ is a settheoretic complete intersection.

(3) If $I$ is a local complete intersection ideal with $\operatorname{dim}(A / I) \geq 1$, then $I$ is set-theoretically generated by $d-1$ or less elements.

Proof. (1) It follows from a theorem of Katz [Ka] (see the proof of [HS, Theorem 8.73 (2)]) that $I$ has a reduction $J$ such that $J / J^{2}$ is generated by $d-1$ elements. Since $J$ is a reduction of $I$, it is easy to see that $\sqrt{J}=\sqrt{I}$ and $h t(J)=\operatorname{ht}(I)$. If $d \geq 4$, applying (4.4) we see that $J$ is generated by $d-1$ elements. Therefore, $I$ is set-theoretically generated by $d-1$ elements. In the case $d=3$, we apply (3.1).

(2) We have $\operatorname{ht}(I)=d-2$. If $\operatorname{ht}(I)=1$, the result easily follows. Therefore, let $\operatorname{ht}(I) \geq 2$. It follows from the famous Ferrand-Szipro construction that there exists an ideal $J \subset A$ such that $\sqrt{I}=\sqrt{J}$ and $J / J^{2}$ is a free $A / J$-module of rank $d-2$. In the cases when (4.2) is applicable on $J$, we may appeal to (4.2) and conclude that $J$ is a complete intersection. Otherwise, let $J=\left(a_{1}, \cdots, a_{d-2}\right)+J^{2}$ and consider $J_{1}=\left(a_{1}, \cdots, a_{d-3}\right)+J^{(d-3) !}$. Then $\sqrt{J_{1}}=\sqrt{J}(=\sqrt{I})$ and $J_{1}=$ $\left(a_{1}, \cdots, a_{d-3}, a_{d-2}^{(d-3) !}\right)+J_{1}^{2}$. It follows from an argument of Boratynski that $J_{1}$ is a surjective image of a projective $A$-module $P$ of rank $d-2$. By [Ra1, $P$ is free. Therefore, $J_{1}$ is generated by $d-2$ elements and we are done.

(3) This can be proved by induction on $\operatorname{dim}(A / I)$. The case $\operatorname{dim}(A / I)=1$ has been covered in (2). The rest of the argument is standard and uses an induction lemma of Lyubeznik [Ly, 4.2].

Remark 4.7. Recall that the Bass-Quillen conjecture asks whether all projective $S[T]$-modules are free, where $S$ is a regular local ring. It is not hard to see that a positive answer to Quillen's question implies the validity of the Bass-Quillen conjecture. The following corollary is an analogue of the Bass-Quillen conjecture in the geometric case and, as expected, can be deduced from (4.3) which is an analogue of Quillen's question.

Corollary 4.8. Let $S$ be a regular $k$-spot of dimension d with infinite residue field and $n$ be an integer such that $2 n \geq d+3$. Let $I \subset S[T]$ be an ideal of height $n$ such that $I=\left(F_{1}, \cdots, F_{n}\right)+I^{2}$. Then there exist $G_{1}, \cdots, G_{n} \in I$ such that $I=\left(G_{1}, \cdots, G_{n}\right)$, where $G_{i}-F_{i} \in I^{2}$ for $1 \leq i \leq n$.

Proof. Let $\mathfrak{n}$ be the maximal ideal of $S$. Let $R$ denote the local ring obtained from $S\left[T^{-1}\right]$ by localizing at the maximal ideal $\mathfrak{m}=\left(\mathfrak{n}, T^{-1}\right)$ and let $f=T^{-1}$. Then since $S$ is regular, $R$ is a regular local ring (of dimension $d+1$ ) and $f$ is a regular parameter of $R$. Moreover, $R_{f}=S(T)$, where $S(T)$ is the ring obtained from $S[T]$ by inverting all the monic polynomials.

We have $I S(T)=\left(F_{1}, \cdots, F_{n}\right)+I^{2} S(T)$. Since $S(T)$ is of the form $R_{f}$, it follows from (4.3) that there exist $H_{1}, \cdots, H_{n} \in I S(T)$ such that $I S(T)=\left(H_{1}, \cdots, H_{n}\right)$ with $H_{i}-F_{i} \in I^{2} S(T)$. Since $S$ is local, the conclusion of the corollary now follows from [BK, 4.6]. 
The following corollary is an improvement of [MV, Theorem 4]. The proof is the same as that of (4.8).

Corollary 4.9. Let $(S, \mathfrak{m})$ be a regular $k$-spot of dimension $d$ with infinite residue field and $n$ be an integer such that $2 n \geq d+3$. Let $I \subset S[T]$ be an ideal of height $n$ such that $I=\left(F_{1}, \cdots, F_{n}\right)+\left(I^{2} T\right)$. Then there exist $G_{1}, \cdots, G_{n} \in I$ such that $I=\left(G_{1}, \cdots, G_{n}\right)$, where $G_{i}-F_{i} \in\left(I^{2} T\right)$ for $1 \leq i \leq n$.

Before proceeding further, we need to recall a result (Theorem4.10 below). First, we fix some notation.

Let $A$ be a Noetherian ring of dimension $d$ and $n$ be an integer such that $2 n \geq$ $d+3$. Let $I \subset A[T]$ be an ideal of height $n$ such that there is a surjection $\alpha$ : $A[T]^{n} \rightarrow I /\left(I^{2} T\right)$. Now consider the set

$$
N(I ; \alpha)=\left\{s \in A \mid \exists \theta: A_{s}[T]^{n} \rightarrow I_{s} \text { such that } \theta \text { lifts } \alpha_{s}\right\} .
$$

We now quote the following result due to Bhatwadekar and Keshari [BK].

Theorem 4.10. Let $A$ be a regular domain of dimension $d$ containing a field and $n$ be an integer such that $2 n \geq d+3$. Let $I \subset A[T]$ be an ideal of height $n$ such that there is a surjection $\alpha: A[T]^{n} \rightarrow I /\left(I^{2} T\right)$. Then $N(I ; \alpha)$ is an ideal of $A$.

Remark 4.11. The above theorem, in the form quoted here, has been presented in DS] where we called $N(I ; \alpha)$ the Nori ideal of the pair $(I ; \alpha)$. The proof is implicit in the proof of [BK, Theorem 4.13].

The next result answers a question of Nori in a little more generality than BK] (we will prove another version in (5.5)). This theorem is the "global" version of (4.9) and can be easily deduced from (4.9) and (4.10) using a standard local-global argument.

Theorem 4.12. Let $A$ be a regular ring of dimension $d$ which is essentially of finite type over a field $k$ such that $A$ has infinite residue fields and let $n$ be an integer such that $2 n \geq d+3$. Let $I \subset A[T]$ be an ideal of height $n$ such that $I=$ $\left(F_{1}, \cdots, F_{n}\right)+\left(I^{2} T\right)$. Then there exist $G_{1}, \cdots, G_{n} \in I$ such that $I=\left(G_{1}, \cdots, G_{n}\right)$, where $G_{i}-F_{i} \in\left(I^{2} T\right)$ for $1 \leq i \leq n$.

We now consider ideals in a polynomial extension of $R_{f_{1} \cdots f_{t}}$.

Theorem 4.13. Let $k$ be an infinite field and $(R, \mathfrak{m})$ be a regular $k$-spot of dimension $d$. Let $n$ be an integer such that $2 n \geq d+2$. Let $f_{1}, \cdots, f_{t} \in R$ be regular parameters of $R$ (where $t \leq n-2$ ), which are linearly independent modulo $\mathfrak{m}^{2}$. Let $I \subset R_{f_{1} \cdots f_{t}}[X]$ be an ideal of height $n$ such that $I=\left(F_{1}, \cdots, F_{n}\right)+I^{2}$. Then there exist $G_{1}, \cdots, G_{n} \in I$ such that $I=\left(G_{1}, \cdots, G_{n}\right)$, where $G_{i}-F_{i} \in I^{2}$ for $1 \leq i \leq n$.

Proof. Since $k$ is infinite, we can find $\lambda \in k$ such that $I(\lambda)$ is either an ideal of $R_{f_{1} \cdots f_{t}}$ of height $n$ or $I(\lambda)=R_{f_{1} \cdots f_{t}}$. Changing $X$ to $X-\lambda$ we may assume that $I(0)$ is either of height $n$ or $I(0)=R_{f_{1} \cdots f_{t}}$.

We first assume that $I(0)$ is a proper ideal. Applying (4.2) it follows that there exist $a_{1}, \cdots, a_{n} \in I(0)$ such that $I(0)=\left(a_{1}, \cdots, a_{n}\right)$, where $a_{i}-F_{i}(0) \in I(0)^{2}$. We can then apply [BS1, 3.9] and find $H_{1}, \cdots, H_{n} \in I$ such that $I=\left(H_{1}, \cdots, H_{n}\right)+$ $\left(I^{2} X\right)$ such that $H_{i}-F_{i} \in I^{2}$. On the other hand, if $I(0)=R_{f_{1} \cdots f_{t}}$, then we can find such $H_{1}, \cdots, H_{n}$ as well.

Let $\mathfrak{n}$ be any maximal ideal of $R_{f_{1} \cdots f_{t}}$. Then $\left(R_{f_{1} \cdots f_{t}}\right)_{\mathfrak{n}}=R_{\mathfrak{n}}$ and $R_{\mathfrak{n}}$ is a regular local domain of dimension $d-1$ which is essentially of finite type over $k$. Applying 
(4.9) it follows that $I_{\mathfrak{n}}=\left(\beta_{1}, \cdots, \beta_{n}\right)$ such that $\beta_{i}-H_{i} \in\left(I^{2} X\right)_{\mathfrak{n}}$. Now using (4.10) and a local-global argument, the result easily follows.

4.1. The semilocal case. We now briefly indicate how our main results can be generalized to a semilocal regular domain $R$ (with infinite residue fields) which is essentially of finite type over an infinite perfect field $k$. Instead of repeating the proof here, we shall only give an outline. The recipe being the same, a diligent reader should be able to bake a proof in this case.

We start with two easy lemmas. The proofs are left to the reader.

Lemma 4.14. Let $R$ be a regular semilocal domain of dimension $d$ with maximal ideals $\mathfrak{m}_{1}, \cdots, \mathfrak{m}_{r}$ such that $h t\left(\mathfrak{m}_{i}\right)=d$ for $1 \leq i \leq r$. Let $J$ denote the Jacobson radical of $R$. Then there exists $f_{1}, \cdots, f_{d} \in J$ such that:

(1) $J=\left(f_{1}, \cdots, f_{d}\right)$.

(2) The images of $f_{1}, \cdots, f_{d}$ in $R_{\mathfrak{m}_{i}}$ form a system of regular parameters for each $i \in\{1, \cdots, r\}$.

Lemma 4.15. Let $A$ be a semilocal ring and let $J$ be its Jacobson radical. Let $X$ be an indeterminate. Let $F \in A[X]$ be a monic polynomial of the form $F(X)=X^{l}+$ $a_{1} X^{l-1}+\cdots+a_{l}$, where $a_{i} \in J$. (We may call such a polynomial a semi-Weierstrass polynomial.) Then $A[X] \hookrightarrow A[X]_{1+(J, X)}=A[X]_{1+X A[X]}$ is analytically isomorphic along $F$.

We shall have the following setup for the rest of this section.

Assumption 4.16. Let $A$ be a regular affine domain of dimension d over an infinite perfect field $k$. Let $\mathfrak{m}_{1}, \cdots, \mathfrak{m}_{r}$ be maximal ideals of $A$ and let $R=T^{-1} A$, where $T=A \backslash \bigcup_{i=1}^{r} \mathfrak{m}_{i}$. Let $J$ denote the Jacobson radical of $R$. For $1 \leq t \leq d-1$, let $f_{1}, \cdots, f_{t} \in J$ be such that the images of $f_{1}, \cdots, f_{t}$ in $R_{\mathfrak{m}_{i}}$ form a part of a regular system of parameters for each $i \in\{1, \cdots, r\}$.

The following result is a variant of Nashier's result (2.6), as stated in [BS1, 2.12]. A much more general result of a similar flavour can be found in Ga2, CHK.

Theorem 4.17. Let $R, J, f_{1}, \cdots f_{t}$ be as in 4.16 and let $g \in J^{2}$ be such that $\left(g, f_{1}, \cdots f_{t}\right)$ is an $R_{\mathfrak{m}_{j}}$-sequence for each $1 \leq j \leq r$ and $1 \leq t \leq d-1$. Then there exists a regular semilocal ring (subring of $R$ ) such that

(1) $S=k\left[Z_{1}, \cdots, Z_{d}\right]_{1+J^{\prime}}$, where $J^{\prime}=k\left[Z_{1}, \cdots, Z_{d}\right] \cap J$.

(2) $k\left[Z_{1}, \cdots, Z_{d}\right] \cap \mathfrak{m}_{j}=\left(Z_{1}, \cdots, Z_{d-1}, \phi_{j}\left(Z_{d}\right)\right)$ for $1 \leq j \leq r$, where $\phi_{j}\left(Z_{d}\right) \in$ $k\left[Z_{d}\right]$ are distinct irreducible monic polynomials.

(3) $S \hookrightarrow R$ is an analytic isomorphism along $h$ for some $h \in g R \cap S$.

(4) We may assume $Z_{i}=f_{i}$ for $1 \leq i \leq t$.

The semilocal version of (4.2) is as follows.

Theorem 4.18. Let $R, J$ be as in (4.16). Let $n$ be an integer such that $2 n \geq d+2$ and let $I \subset R_{f_{1} \cdots f_{t}}$ be an ideal of height $n$, where $t \leq n-2$ and $f_{1}, \cdots, f_{t} \in J$ form a part of a regular system of parameters of $R_{\mathfrak{m}_{j}}$ for each $j \in\{1, \cdots, r\}$. Suppose it is given that $I=\left(a_{1}, \cdots, a_{n}\right)+I^{2}$. Then there exists $b_{1}, \cdots, b_{n}$ such that $I=\left(b_{1}, \cdots, b_{n}\right)$, where $b_{i}-a_{i} \in I^{2}$. 


\section{THE CASE OF DVR}

We shall use the following theorem of S. P. Dutta to prove our next set of results. This result of Dutta is an improvement of Nashier's result stated as Theorem 2.6 in this paper.

Theorem 5.1 ( $\overline{\mathrm{Du}}$, Theorem 1.3]). Let $(R, \mathfrak{m}, k)$ be a regular local ring of dimension $d+1$, essentially of finite type and smooth over an excellent $D V R(V, \pi)$ such that $k$ is infinite and is separably generated over $V / \pi V$. Let $a \in \mathfrak{m}^{2}$ be such that $a \notin \pi R$ (i.e., $\{\pi, a\}$ form an $R$-sequence). Assume further that $\left(a, f_{1}, \cdots, f_{t}\right)$ is a sequence in $R$, where $1 \leq t \leq d-1$, with $f_{1}, \cdots, f_{t}$ part of a minimal set of generators of $\mathfrak{m}$ modulo $\mathfrak{m}^{2}$. Then there exists a regular local ring $(S, \mathfrak{n}, k) \subset(R, \mathfrak{m}, k)$ such that

(1) $S=W\left[Z_{1}, \cdots, Z_{d}\right]_{\left(\pi, Z_{1}, Z_{2}, \cdots, \phi\left(Z_{d}\right)\right)}$, where $\phi$ is a monic irreducible polynomial in $W\left[Z_{d}\right]$ and $(W, \pi)$ is an excellent DVR contained in $R$. Moreover, $R$ is an étale neighbourhood of $S$.

(2) We may assume that $Z_{i}=f_{i}$ for $1 \leq i \leq t$.

(3) There exists an element $h \in S \cap a R$ such that $S / h S \longrightarrow R / a R$ is an isomorphism. Furthermore, $h R=a R$.

Theorem 5.2. Let $(R, \mathfrak{m}, k)$ be a regular local ring of dimension $d+1$, essentially of finite type and smooth over an excellent $D V R(V, \pi)$ such that $k$ is infinite and is separably generated over $V / \pi V$. Let $n$ be an integer such that $2 n \geq d+3$. Let $f_{1}, \cdots, f_{t} \in R$ be regular parameters of $R$ (where $\left.t \leq n-2\right)$. Let $I \subset R_{f_{1} \cdots f_{t}}$ be an ideal of height $n$. Suppose it is given that $I=\left(a_{1}, \cdots, a_{n}\right)+I^{2}$. Then there exist $b_{1}, \cdots, b_{n} \in I$ such that $I=\left(b_{1}, \cdots, b_{n}\right)$, where $b_{i}-a_{i} \in I^{2}$ for $1 \leq i \leq n$.

Proof. The proof is along similar lines as (4.2), with some subtle modifications. Details of these modifications will be discussed, but for other portions we shall only give a sketch.

First of all, if one of $f_{1}, \cdots, f_{t}$ is $\pi$, we are reduced to the field case (4.2). Therefore, we assume that none of $f_{1}, \cdots, f_{t}$ is $\pi$.

Consider $J=I \cap R$. We have $\operatorname{ht}(J)=n \geq t+2$. Since both $\pi R$ and $\left(f_{1}, \cdots, f_{t}\right) R$ are prime ideals of $R$, we can find $a \in J \cap \mathfrak{m}^{2}$ such that $a \notin \pi R$ and $a \notin\left(f_{1}, \cdots, f_{t}\right) R$. Therefore, $\left(f_{1}, \cdots, f_{t}, a\right)$ is an $R$-sequence, and since $R$ is local, $\left(a, f_{1}, \cdots, f_{t}\right)$ is also an $R$-sequence. By (5.1), there exists a regular local $\operatorname{ring}(S, \mathfrak{n}, k) \subset(R, \mathfrak{m}, k)$ such that $S=W\left[Z_{1}, \cdots, Z_{d}\right]_{\left(\pi, Z_{1}, Z_{2}, \cdots, \phi\left(Z_{d}\right)\right)}$, where $\phi$ is a monic irreducible polynomial in $W\left[Z_{d}\right]$ and $(W, \pi)$ is an excellent DVR contained in $R$. Moreover, $S \hookrightarrow R$ is analytically isomorphic along $h$ for some $h \in a R \cap S$. Also, we may assume that $f_{i}=Z_{i}$ for $i=1, \cdots, t$. Therefore, $S_{Z_{1} \cdots Z_{t}} \hookrightarrow R_{f_{1} \cdots f_{t}}$ is analytically isomorphic along $h$. Let $I_{1}=I \cap S_{Z_{1} \cdots Z_{t}}$. Then $I_{1} / I_{1}^{2} \simeq I / I^{2}$ and as in (4.2) it is enough to prove the theorem for $I_{1}$ and $S_{Z_{1} \cdots Z_{t}}$, in place of $I$ and $R_{f_{1} \cdots f_{t}}$.

Suppose that we have $I_{1}=\left(\alpha_{1}, \cdots, \alpha_{n}\right)+I_{1}^{2}$. Let 'bar' denote reduction modulo the ideal $(\pi)$. Then $\operatorname{ht}\left(\overline{I_{1}}\right) \geq n-1$ and we have $\overline{I_{1}}=\left(\overline{\alpha_{1}}, \cdots, \overline{\alpha_{n}}\right)+{\overline{I_{1}}}^{2}$. There exists $\bar{s} \in{\overline{I_{1}}}^{2}$ such that $\overline{I_{1}}=\left(\overline{\alpha_{1}}, \cdots, \overline{\alpha_{n}}, \bar{s}\right)$. Adding suitable multiples of $\bar{s}$ to $\overline{\alpha_{1}}, \cdots, \overline{\alpha_{n}}$, if necessary, we can conclude by [EE, Theorem $\left.\mathrm{A}\right]$ that there is an ideal $L \subseteq \bar{S}_{Z_{1} \cdots Z_{t}}$ such that: (1) $\left(\overline{\beta_{1}}, \cdots, \overline{\beta_{n}}\right)=L \cap \overline{I_{1}}$, where $\overline{\beta_{i}}-\overline{\alpha_{i}} \in{\overline{I_{1}}}^{2} ;$ (2) $\operatorname{ht}(L) \geq n ;(3) L+\overline{I_{1}}=\bar{S}_{Z_{1} \cdots Z_{t}}$.

Let us first assume that $L$ is proper and $\operatorname{ht}(L)=n$. We have $L=\left(\overline{\alpha_{1}}, \cdots, \overline{\alpha_{n}}\right)+$ $L^{2}$. We now note that $\bar{S}=k\left[Z_{1}, \cdots, Z_{d}\right]_{\left(Z_{1}, Z_{2}, \cdots, \phi\left(Z_{d}\right)\right)}$, where $k$ is the residue 
field of $W$, which is the same as the residue field of $R$ and is infinite. Therefore, applying (4.2), we conclude that $\overline{\alpha_{1}}, \cdots, \overline{\alpha_{n}}$ can be lifted to a set of $n$ generators of $L$. We can now apply [DS, Proposition 3.3] to see that there exist $\overline{\gamma_{1}}, \cdots, \overline{\gamma_{n}}$ such that $\overline{I_{1}}=\left(\overline{\gamma_{1}}, \cdots, \overline{\gamma_{n}}\right)$, where $\overline{\gamma_{i}}-\overline{\alpha_{i}} \in{\overline{I_{1}}}^{2}$. We note that such a conclusion holds trivially if $L$ is not proper. As a consequence, we have $I_{1}=\left(\gamma_{1}, \cdots, \gamma_{n}\right)+I_{1}^{2} \cap(\pi)$.

There exists $c \in I_{1}^{2} \cap(\pi)$ such that $I_{1}=\left(\gamma_{1}, \cdots, \gamma_{n}, c\right)$. Adding suitable multiples of $c$ to $\gamma_{1}, \cdots, \gamma_{n}$ if necessary, we may apply [EE, Theorem A] and see that there exists an ideal $M \subseteq S_{Z_{1} \cdots Z_{t}}$ such that: (1) $I_{1} \cap M=\left(\delta_{1}, \cdots, \delta_{n}\right)$, where $\delta_{i}-\gamma_{i} \in I_{1}^{2}$; (2) $\operatorname{ht}(M) \geq n ;(3) M+I_{1}^{2} \cap(\pi)=S_{Z_{1} \cdots Z_{t}}$.

We note that $M=\left(\delta_{1}, \cdots, \delta_{n}\right)+M^{2}$, and in view of [BS3, Subtraction principle], it is enough to show that $\delta_{1}, \cdots, \delta_{n}$ can be lifted to a set of generators of $M$.

We consider the ideal $N=M \cap W\left[Z_{t}, \cdots, Z_{d-1}\right]$. Then $\operatorname{ht}(N) \geq n-t \geq 2$. By [Na, Proposition 1.11], $N$ contains a non-zero form $\widetilde{F}$ in $W\left[Z_{t}, \cdots, Z_{d-1}\right]$. We may write $\widetilde{F}=\pi^{l} F^{\prime}$, where $l \geq 0$ and $F^{\prime} \notin \pi W\left[Z_{t}, \cdots, Z_{d-1}\right]$. We may assume that $Z_{t}$ does not divide $F^{\prime}$ in $W\left[Z_{t}, \cdots, Z_{d}\right]$. Now $M$ contains $\widetilde{F}=\pi^{l} F^{\prime}$. Let $\mathfrak{P}$ be any prime ideal of $S_{Z_{1} \cdots Z_{t}}$ containing $M$. Since $M+\pi S_{Z_{1} \cdots Z_{t}}=S_{Z_{1} \cdots Z_{t}}$, it follows that $F^{\prime} \in \mathfrak{P}$. Therefore, $\sqrt{M}$ contains $F^{\prime}$ and, consequently, $M$ contains a power of $F^{\prime}$, say $F$, which still is a form in $W\left[Z_{t}, \cdots, Z_{d-1}\right]$. Therefore $F \in$ $M \cap W\left[Z_{t}, \cdots, Z_{d-1}\right]=N$. Since $F \notin \pi W\left[Z_{t}, \cdots, Z_{d-1}\right]$, by [Na, Remark 1.14] we conclude that $F$ represents a unit in $W$. We can now make a homogeneous change of variables (see Remark 2.5) and assume that $F$ is a Weierstrass polynomial in one of the variables $Z_{t+1}, \cdots, Z_{d-1}$. The rest of the proof is exactly the same as (4.2).

Following the same method as in (4.8) we can prove:

Corollary 5.3. Let $(A, \mathfrak{m}, k)$ be a regular local ring of dimension d, essentially of finite type and smooth over an excellent $D V R(V, \pi)$ such that $A / \mathfrak{m}$ is infinite and is separably generated over $V / \pi V$. Let $n$ be an integer such that $2 n \geq d+3$. Let $I \subset A[T]$ be an ideal of height $n$ such that $I=\left(f_{1}, \cdots, f_{n}\right)+I^{2}$. Then there exist $g_{1}, \cdots, g_{n} \in I$ such that $I=\left(g_{1}, \cdots, g_{n}\right)$, where $g_{i}-f_{i} \in I^{2}$ for $1 \leq i \leq n$.

As $A$ is local, it is easy to deduce the following (compare it with (4.9)):

Corollary 5.4. Let $A, I, d, n$ be as above. Suppose it is given that $I=\left(f_{1}, \cdots, f_{n}\right)+$ $\left(I^{2} T\right)$. Then there exist $g_{1}, \cdots, g_{n} \in I$ such that $I=\left(g_{1}, \cdots, g_{n}\right)$, where $g_{i}-f_{i} \in$ $\left(I^{2} T\right)$ for $1 \leq i \leq n$.

We remark that (4.10) is true for the type of rings we are considering in this section. If one examines the proof of (4.10) (either from [BK] or DS]), one can see that the hypothesis "regular domain containing a field" is only used to ensure patching where a certain unimodular row over $B[T]$ (where $B$ is a regular local ring containing a field) is required to be completable. But this is also true for the "unramified" case we are considering here (see $\mathrm{PO}$ ). Therefore, we obtain the following theorem, which answers a question of Nori (see [BK] for smooth finitely generated algebras. Note that for any prime $p \in \mathbb{Z}$, the ring $\mathbb{Z}_{(p)}$ is an excellent DVR.

Theorem 5.5. Let $A$ be regular domain, which is smooth and essentially of finite type over $\mathbb{Z}$, with infinite residue fields. Let $\operatorname{dim} A=d$ and let $n$ be an integer such 
that $2 n \geq d+3$. Let $I \subset A[T]$ be an ideal of height $n$ such that $I=\left(f_{1}, \cdots, f_{n}\right)+$ $\left(I^{2} T\right)$. Then there exist $g_{1}, \cdots, g_{n} \in I$ such that $I=\left(g_{1}, \cdots, g_{n}\right)$, where $g_{i}-f_{i} \in$ $\left(I^{2} T\right)$ for $1 \leq i \leq n$.

\section{ACKNOWLEDGEMENTS}

The author is indebted to S. M. Bhatwadekar for kindly sharing his ideas. The author sincerely thanks S. M. Bhatwadekar and Ravi Rao for always answering all queries, patiently and promptly. The author sincerely thanks the referee for going through the manuscript with great care. A detailed list of corrections and suggestions by the referee improved the exposition significantly.

\section{REFERENCES}

[Bh] S. M. Bhatwadekar, Cancellation theorems for projective modules over a two dimensional ring and its polynomial extensions, Compositio Math. 128 (2001), 339-359. MR1858341 (2002h:13016)

[BK] S. M. Bhatwadekar and M. K. Keshari, A question of Nori: Projective generation of ideals, K-Theory 28 (2003), 329-351. MR2017619 (2004j:13013)

[BR] S. M. Bhatwadekar and Ravi A. Rao, On a question of Quillen, Trans. Amer. Math. Soc. 279 (1983), 801-810. MR709584 (85g:13003)

[BS1] S. M. Bhatwadekar and Raja Sridharan, Projective generation of curves in polynomial extensions of an affine domain and a question of Nori, Invent. Math. 133 (1998), 161-192. MR.1626485 (99c:13016)

[BS2] S. M. Bhatwadekar and Raja Sridharan, The Euler class group of a Noetherian ring, Compositio Math. 122 (2000), 183-222. MR1775418 (2001g:13018)

[BS3] S. M. Bhatwadekar and Raja Sridharan, On Euler classes and stably free projective modules, in: Algebra, arithmetic and geometry, Part I, II (Mumbai, 2000), 139-158, Tata Inst. Fund. Res. Stud. Math., 16, Tata Inst. Fund. Res., Bombay, 2002. MR1940666 (2003i:13012)

[CHK] Jean-Louis Colliot-Thélène, Raymond T. Hoobler and Bruno Kahn, The Bloch-OgusGabber theorem, in: Algebraic $K$-theory (Toronto, ON, 1996), 31-94, Fields Inst. Commun., 16, Amer. Math. Soc., Providence, RI, 1997. MR.1466971 (98j:14021)

[Da1] M. K. Das, The Euler class group of a polynomial algebra, J. Algebra 264 (2003), 582-612. MR1981423 (2004c:13031)

[Da2] M. K. Das, The Euler class group of a polynomial algebra II, J. Algebra 299 (2006), 94-114. MR2225766 (2007a:13023)

[DS] M. K. Das and Raja Sridharan, Euler class groups and a theorem of Roitman, J. Pure and Appl. Algebra 215 (2011), 1340-1347. MR2769235(2012c:13022)

$[\mathrm{Du}]$ S. P. Dutta, A theorem on smoothness-Bass-Quillen, Chow groups and intersection multiplicity of Serre, Trans. Amer. Math. Soc. 352 (1999), 1635-1645. MR1621737 (2000i:13016)

[EE] D. Eisenbud and E. G. Evans, Generating modules efficiently: Theorems from algebraic K-Theory, J. Algebra 27 (1973), 278-305. MR0327742 (48:6084)

[Ga1] O. Gabber, "Some theorems on Azumaya algebras" in The Brauer Group (Sem., Les Plans-sur-Bex, 1980), Lecture Notes in Math., 844, Springer, Berlin-New York, 1981, 129-209. MR611868 (83d:13004)

[Ga2] O. Gabber, Gersten's conjecture for some complexes of vanishing cycles, Manuscripta Math. 85 (1994), 323-343. MR1305746 (96m:14010)

[HS] C. Huneke and I. Swanson, Integral closure of ideals, rings, and modules, in: London Mathematical Society Lecture Note Series, Vol. 336, Cambridge University Press, Cambridge, 2006. MR2266432(2008m:13013)

[Ka] D. Katz, Generating ideals up to projective equivalence, Proc. Amer. Math. Soc. 120 (1994), 401-414. MR1176070 (94b:13013)

[Li] H. Lindel, On the Bass-Quillen conjecture concerning projective modules over polynomial rings, Invent. Math. 65 (1981), 319-323. MR641133 (83g:13009) 
[Ly] G. Lyubeznik, The number of defining equations of affine algebraic sets, Amer. J. Math. 114 (1992), 413-463. MR 1156572 (93b:13018)

[Ma] S. Mandal, On efficient generation of ideals, Invent. Math. 75 (1984), 59-67. MR728138 (85d:13017)

[MV] S. Mandal and P. L. N. Varma, On a question of Nori: The local case, Communications in Algebra 25 (1997), 451-457. MR1428789 (98a:13026)

[MMR] N. Mohan Kumar, M. P. Murthy and Amit Roy, A cancellation theorem for projective modules over finitely generated rings, in: Algebraic geometry and commutative algebra, Vol. I, 281-287, Kinokuniya, Tokyo, 1988. MR977765 (89j:18009)

$[\mathrm{Mu}] \quad$ M. P. Murthy, Projective $A[X]$-modules, J. London Math. Soc., 41 (1966), 453-456. MR0200289 (34:188)

[Na] B. S. Nashier, Efficient generation of ideals in polynomial rings, Journal of Algebra 85 (1983), 287-302. MR725083 (85f:13010)

[Ni] Y. Nisnevich, Stratified canonical forms of matrix valued functions in a neighborhood of a transition point, Internat. Math. Res. Notices. 10 (1998), 513-527. MR 1634912 (2000c:32082)

[Po] D. Popescu, Polynomial rings and their projective modules, Nagoya Math. J. 113 (1989), 121-128. MR986438 (90g:13017)

[Qu] D. Quillen, Projective modules over polynomial rings, Invent. Math. 36 (1976), 167-171. MR0427303 (55:337)

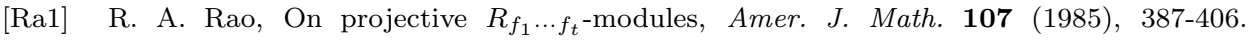
MR784289 (86j:13011)

[Ra2] R. A. Rao, The Bass-Quillen conjecture in dimension three but characteristic $\neq 2,3$ via a question of A. Suslin, Invent. Math. 93 (1988), no. 3, 609-618. MR952284 (89d:13011)

[S] Raja Sridharan, Projective modules and complete intersections, K-Theory 13 (1998), 269-278. MR.1609901 (99c:13019)

[SV] A. A. Suslin and L. N. Vaserstein, Serre's problem on projective modules over polynomial rings and algebraic K-theory, Izv. Akad. Nauk. SSSR, Ser. Mat. 40 (1976), 937-1001. MR0447245 (56:5560)

[Sw1] R. G. Swan, Vector bundles, projective modules and the $K$-theory of spheres, in: Algebraic topology and algebraic K-theory (Princeton, N.J., 1983), Ann. of Math. Studies, Vol. 113, Princeton Univ. Press, Princeton, NJ, 1987, 432-522. MR921488 (89f:18009)

[Sw2] R. G. Swan, Néron-Popescu desingularization, in: Algebra and Geometry (Taipei, 1995), Lect. Algebra Geom., Vol. 2, International Press, Cambridge, MA, 135-192. MR.1697953 (2000h:13006)

Stat-Math Unit, Indian Statistical Institute, 203 B. T. Road, Kolkata 700108 India

E-mail address: mrinal@isical.ac.in 